\title{
Sequential fractional differential equations and unification of anti-periodic and multi-point boundary conditions
}

\author{
Ahmed Alsaedi, Bashir Ahmad*, Mohammed H. Aqlan \\ Nonlinear Analysis and Applied Mathematics (NAAM)-Research Group, Department of Mathematics, Faculty of Science, King Abdulaziz \\ University, P. O. Box 80203, Jeddah 21589, Saudi Arabia.
}

Communicated by N. Shahzad

\begin{abstract}
In this paper, we present a novel idea of unification of anti-periodic and multi-point boundary conditions and develop the existence theory for sequential fractional differential equations supplemented with these new conditions. We apply fixed point theorems due to Banach, Krasnoselskii, Leray-Schauder alternative criterion, and Leray-Schauder degree theory to obtain the desired results. Our results are well-illustrated with the aid of examples and correspond to some new special cases for particular choices of parameters involved in the problem. (C)2017 All rights reserved.
\end{abstract}

Keywords: Sequential fractional differential equations, nonlocal, anti-periodic, multi-point, existence, fixed point. 2010 MSC: 34A08, 34B10, 34B15.

\section{Introduction}

In this paper, we study a nonlinear anti-periodic type multi-point boundary value problems of sequential fractional differential equations given by

$$
\left\{\begin{array}{c}
\left({ }^{c} D^{q}+k^{c} D^{q-1}\right) u(t)=f(t, u(t)), \quad 2<q \leqslant 3, \quad 0<t<T, \\
\alpha_{1} u(0)+\sum_{i=1}^{m} a_{i} u\left(\eta_{i}\right)+\gamma_{1} u(T)=\beta_{1}, \\
\alpha_{2} u^{\prime}(0)+\sum_{i=1}^{m} b_{i} u^{\prime}\left(\eta_{i}\right)+\gamma_{2} u^{\prime}(T)=\beta_{2} \\
\alpha_{3} u^{\prime \prime}(0)+\sum_{i=1}^{m} c_{i} u^{\prime \prime}\left(\eta_{i}\right)+\gamma_{3} u^{\prime \prime}(T)=\beta_{3}
\end{array}\right.
$$

where ${ }^{c} D^{q}$ denotes the Caputo fractional derivative of order $q, \alpha_{j}, \beta_{j}, \gamma_{j} \in \mathbb{R}(j=1,2,3), a_{i}, b_{i}, c_{i} \in$ $\mathbb{R}(i=1,2, \ldots, m), k \in \mathbb{R}^{+}$and $f$ is an appropriately chosen continuous function. The new boundary

\footnotetext{
*Corresponding author

Email addresses: aalsaedi@hotmail .com (Ahmed Alsaedi), bashirahmad_qau@yahoo.com (Bashir Ahmad), aqlan76@gmail. com (Mohammed H. Aqlan)

doi:10.22436/jnsa.010.01.07
} 
conditions in (1.1) can be interpreted as the values of the unknown function and its first and second-order derivatives at the end points of the interval under consideration relate to the linear combination of the values of the unknown function, and its first and second-order derivatives at interior points $\eta_{i} \in(0, T)$.

There has been a great interest in developing theoretical analysis for a variety of boundary value problems of nonlinear fractional order differential equations in the recent years. Anti-periodic and multi-point boundary value problems are two important classes of such problems, which have received considerable attention. Anti-periodic boundary conditions appear in the mathematical modeling of certain physical processes and phenomena, for example, see [6] and the references cited therein. Nonlocal multi-point conditions are regarded as more plausible than the classical initial/boundary conditions as these condition can describe peculiarities of chemical, physical or other processes happening inside the domain, for instance, see [9]. For some recent works on fractional-order anti-periodic and multi-point boundary value problems, we refer the reader to a series of papers $([1-5,7,8,10,12,14-16,18-20]$ and the references cited therein.

The objective of the present paper is to investigate the existence of solutions for sequential fractional differential equations equipped with a new kind of boundary conditions consisting of a combination of anti-periodic and multi-point boundary conditions. The rest of the paper is organized as follows. Section 2 contains some preliminary concepts of fractional calculus and an auxiliary lemma, which plays a key role in the forthcoming analysis. In Section 3, we prove the uniqueness of solutions for the given problem, while the existence results are presented in Section 4. Illustrative examples are discussed in Section 5. The paper concludes some interesting remarks described in Section 6.

\section{Preliminary work}

First of all, we recall some basic definitions [13, 21].

Definition 2.1. The fractional integral of order $r$ with the lower limit zero for a function $f$ is defined as

$$
I^{r} f(t)=\frac{1}{\Gamma(r)} \int_{0}^{t} \frac{f(s)}{(t-s)^{1-r}} d s, \quad t>0, \quad r>0,
$$

provided the right hand-side is point-wise defined on $[0, \infty)$, where $\Gamma(\cdot)$ is the gamma function, which is defined by $\Gamma(r)=\int_{0}^{\infty} t^{r-1} e^{-t} d t$.

Definition 2.2. The Riemann-Liouville fractional derivative of order $r>0, n-1<r<n, n \in N$, is defined as

$$
D_{0+}^{r} f(t)=\frac{1}{\Gamma(n-r)}\left(\frac{d}{d t}\right)^{n} \int_{0}^{t}(t-s)^{n-r-1} f(s) d s,
$$

where the function $f(t)$ has absolutely continuous derivative up to order $(n-1)$.

Definition 2.3. The Caputo derivative of order $r$ for a function $f:[0, \infty) \rightarrow R$ can be written as

$$
{ }^{c} D^{r} f(t)=D^{r}\left(f(t)-\sum_{k=0}^{n-1} \frac{t^{k}}{k !} f^{(k)}(0)\right), \quad t>0, \quad n-1<r<n .
$$

Remark 2.4. If $\mathrm{f}(\mathrm{t}) \in \mathrm{C}^{\mathfrak{n}}[0, \infty)$, then

$$
{ }^{c} D^{r} f(t)=\frac{1}{\Gamma(n-r)} \int_{0}^{t} \frac{f^{(n)}(s)}{(t-s)^{r+1-n}} d s=I^{n-r} f^{(n)}(t), \quad t>0, \quad n-1<q<n .
$$

To define a solution for the given problem, we need the following lemma. 
Lemma 2.5. Let $\mathrm{h} \in \mathrm{C}[0, \mathrm{~T}], \mathrm{u} \in \mathrm{C}^{3}[0, \mathrm{~T}]$. Then the following linear problem

$$
\left\{\begin{array}{c}
\left({ }^{c} D^{q}+k^{c} D^{q-1}\right) u(t)=h(t), \quad 2<q \leqslant 3, \quad 0<t<T, \\
\alpha_{1} u(0)+\sum_{i=1}^{m} a_{i} u\left(\eta_{i}\right)+\gamma_{1} u(T)=\beta_{1}, \\
\alpha_{2} u^{\prime}(0)+\sum_{i=1}^{m} b_{i} u^{\prime}\left(\eta_{i}\right)+\gamma_{2} u^{\prime}(T)=\beta_{2}, \\
\alpha_{3} u^{\prime \prime}(0)+\sum_{i=1}^{m} c_{i} u^{\prime \prime}\left(\eta_{i}\right)+\gamma_{3} u^{\prime \prime}(T)=\beta_{3},
\end{array}\right.
$$

is equivalent to the fractional integral equation

$$
\begin{aligned}
u(t)= & v_{1}(t)+\int_{0}^{t} e^{-k(t-s)}\left(\int_{0}^{s} \frac{(s-x)^{q-2}}{\Gamma(q-1)} h(x) d x\right) d s \\
& +\sum_{i=1}^{m} \omega_{i}(t) \int_{0}^{\eta_{i}} e^{-k\left(\eta_{i}-s\right)}\left(\int_{0}^{s} \frac{(s-x)^{q-2}}{\Gamma(q-1)} h(x) d x\right) d s+v_{2}(t) \int_{0}^{T} e^{-k(T-s)}\left(\int_{0}^{s} \frac{(s-x)^{q-2}}{\Gamma(q-1)} h(x) d x\right) d s \\
& +\sum_{i=1}^{m} \psi_{i}(t) \int_{0}^{\eta_{i}} \frac{\left(\eta_{i}-s\right)^{q-2}}{\Gamma(q-1)} h(s) d s+v_{3}(t) \int_{0}^{T} \frac{(T-s)^{q-2}}{\Gamma(q-1)} h(s) d s \\
& +\sum_{i=1}^{m} \varphi_{i}(t) \int_{0}^{\eta_{i}} \frac{\left(\eta_{i}-s\right)^{q-3}}{\Gamma(q-2)} h(s) d s+v_{4}(t) \int_{0}^{T} \frac{(T-s)^{q-3}}{\Gamma(q-2)} h(s) d s
\end{aligned}
$$

where

$$
\begin{aligned}
v_{1}(t)= & \frac{\beta_{1}}{\lambda_{1}}-\left(\frac{\sum_{i=1}^{m} a_{i} \eta_{i}+\gamma_{1} T}{\lambda_{1} \lambda_{2}}\right) \beta_{2}-\frac{\beta_{3}}{k^{2} \lambda_{1} \lambda_{2} \delta_{3}}\left(\lambda_{2} \delta_{1}+k \delta_{2}\left(\sum_{i=1}^{m} a_{i} \eta_{i}+\gamma_{1} T\right)\right)+\frac{\beta_{3} e^{-k t}}{k^{2} \delta_{3}} \\
& +\frac{t}{\lambda_{2}}\left(\beta_{2}+\frac{\beta_{3} \delta_{2}}{k \delta_{3}}\right), \\
\omega_{i}(t)= & \frac{c_{i} \delta_{1}}{\delta_{3} \lambda_{1}}-\frac{a_{i}}{\lambda_{1}}-\left(\frac{k\left(\sum_{i=1}^{m} a_{i} \eta_{i}+\gamma_{1} T\right)}{\lambda_{1} \lambda_{2} \delta_{3}}\right)\left(b_{i} \delta_{3}-\delta_{2} c_{i}\right)-\frac{c_{i} e^{-k t}}{\delta_{3}}+\frac{k t}{\lambda_{2} \delta_{3}}\left(b_{i} \delta_{3}-\delta_{2} c_{i}\right), \\
v_{2}(t)= & \frac{\gamma_{3} \delta_{1}}{\delta_{3} \lambda_{1}}-\frac{\gamma_{1}}{\lambda_{1}}-\left(\frac{k\left(\sum_{i=1}^{m} a_{i} \eta_{i}+\gamma_{1} T\right)}{\lambda_{1} \lambda_{2} \delta_{3}}\right)\left(\gamma_{2} \delta_{3}-\delta_{2} \gamma_{3}\right)-\frac{\gamma_{3} e^{-k t}}{\delta_{3}}+\frac{k t}{\lambda_{2} \delta_{3}}\left(\gamma_{2} \delta_{3}-\delta_{2} \gamma_{3}\right), \\
\psi_{i}(t)= & \frac{-\delta_{1} c_{i}}{k \lambda_{1} \delta_{3}}-\left(\frac{\sum_{i=1}^{m} a_{i} \eta_{i}+\gamma_{1} T}{\lambda_{1} \lambda_{2} \delta_{3}}\right)\left(\delta_{2} c_{i}-b_{i} \delta_{3}\right)+\frac{c_{i} e^{-k t}}{k \delta_{3}}+\frac{t}{\lambda_{2} \delta_{3}}\left(\delta_{2} c_{i}-b_{i} \delta_{3}\right), \\
v_{3}(t)= & \frac{-\delta_{1} \gamma_{3}}{k \lambda_{1} \delta_{3}}-\left(\frac{\sum_{i=1}^{m} a_{i} \eta_{i}+\gamma_{1} T}{\lambda_{1} \lambda_{2} \delta_{3}}\right)\left(\delta_{2} \gamma_{3}-\gamma_{2} \delta_{3}\right)+\frac{\gamma_{3} e^{-k t}}{k \delta_{3}}+\frac{t}{\lambda_{2} \delta_{3}}\left(\delta_{2} \gamma_{3}-\gamma_{2} \delta_{3}\right), \\
\varphi_{i}(t)= & \frac{c_{i}}{k^{2} \delta_{3} \lambda_{1} \lambda_{2}}\left(\lambda_{2} \delta_{1}+\delta_{2} k\left(\sum_{i=1}^{m} a_{i} \eta_{i}+\gamma_{1} T\right)\right)-\frac{c_{i} e^{-k t}}{k^{2} \delta_{3}}-\frac{c_{i} \delta_{2} t}{k \delta_{3} \lambda_{2}}, \\
v_{4}(t)= & \frac{\gamma_{3}}{k^{2} \delta_{3} \lambda_{1} \lambda_{2}}\left(\lambda_{2} \delta_{1}+\delta_{2} k\left(\sum_{i=1}^{m} a_{i} \eta_{i}+\gamma_{1} T\right)\right)-\frac{\gamma_{3} e^{-k t}}{k^{2} \delta_{3}}-\frac{\gamma_{3} \delta_{2} t}{k \delta_{3} \lambda_{2}}, \\
\delta_{1}= & \alpha_{1}+\sum_{i=1}^{m} a_{i} e^{-k \eta_{i}}+\gamma_{1} e^{-k T}, \quad \delta_{2}=\alpha_{2}+\sum_{i=1}^{m} b_{i} e^{-k \eta_{i}}+\gamma_{2} e^{-k T}, \\
\delta_{3}= & \alpha_{3}+\sum_{i=1}^{m} c_{i} e^{-k \eta_{i}}+\gamma_{3} e^{-k T} \neq 0, \quad \lambda_{1}=\alpha_{1}+\sum_{i=1}^{m} a_{i}+\gamma_{1} \neq 0, \quad \lambda_{2}=\alpha_{2}+\sum_{i=1}^{m} b_{i}+\gamma_{2} \neq 0
\end{aligned}
$$

Proof. We know that the general solution of (2.1) can be expressed in term of an integral equation as 


$$
u(t)=A_{0} e^{-k t}+A_{1}+A_{2} t+\int_{0}^{t} e^{-k(t-s)} I^{q-1} h(s) d s,
$$

where $A_{0}, A_{1}$ and $A_{2}$ are arbitrary constants and

$$
I^{q-1} h(t)=\int_{0}^{t} \frac{(t-x)^{q-2}}{\Gamma(q-1)} h(x) d x .
$$

By differentiating (2.4) with respect to $t$, we obtain

$$
\begin{gathered}
u^{\prime}(t)=-k A_{0} e^{-k t}+A_{2}-k \int_{0}^{t} e^{-k(t-s)} I^{q-1} h(s) d s+I^{q-1} h(t), \\
u^{\prime \prime}(t)=k^{2} A_{0} e^{-k t}+k^{2} \int_{0}^{t} e^{-k(t-s)} I^{q-1} h(s) d s-k I^{q-1} h(t)+I^{q-2} h(t) .
\end{gathered}
$$

By using the boundary conditions given by (2.1) in (2.4), (2.5), (2.6), together with the notations (2.3), we get

$$
\begin{gathered}
\delta_{1} A_{0}+\lambda_{1} A_{1}+A_{2}\left(\sum_{i=1}^{m} a_{i} \eta_{i}+\gamma_{1} T\right)+\sum_{i=1}^{m} a_{i} \int_{0}^{\eta_{i}} e^{-k(\eta-s)} I^{q-1} h(s) d s \\
+\gamma_{1} \int_{0}^{T} e^{-k(T-s)} I^{q-1} h(s) d s=\beta_{1}, \\
-k \delta_{2} A_{0}+\lambda_{2} A_{2}+\sum_{i=1}^{m} b_{i}\left(-k \int_{0}^{\eta_{i}} e^{-k\left(\eta_{i}-s\right)} I^{q-1} h(s) d s+I^{q-1} h\left(\eta_{i}\right)\right) \\
+\gamma_{2}\left(-k \int_{0}^{T} e^{-k(T-s)} I^{q-1} h(s) d s+I^{q-1} h(T)\right)=\beta_{2}, \\
A_{0} k^{2} \delta_{3}+\sum_{i=1}^{m} c_{i}\left(k^{2} \int_{0}^{\eta_{i}} e^{-k\left(\eta_{i}-s\right)} I^{q-1} h(s) d s-k I^{q-1} h\left(\eta_{i}\right)+I^{q-2} h\left(\eta_{i}\right)\right) \\
+\gamma_{3}\left(k^{2} \int_{0}^{T} e^{-k(T-s)} I^{q-1} h(s) d s-k I^{q-1} h(T)+I^{q-2} h(T)\right)=\beta_{3} .
\end{gathered}
$$

By solving the system (2.7), (2.8), (2.9) for $A_{0}, A_{1}$ and $A_{2}$, we obtain

$$
\begin{aligned}
A_{0}= & \frac{1}{k^{2} \delta_{3}}\left\{\beta_{3}-\sum_{i=1}^{m} c_{i}\left(k^{2} \int_{0}^{\eta_{i}} e^{-k\left(\eta_{i}-s\right)} I^{q-1} h(s) d s-k I^{q-1} h\left(\eta_{i}\right)+I^{q-2} h\left(\eta_{i}\right)\right)\right. \\
& \left.-\gamma_{3}\left(k^{2} \int_{0}^{T} e^{-k(T-s)} I^{q-1} h(s) d s-k I^{q-1} h(T)+I^{q-2} h(T)\right)\right\}, \\
A_{1}= & \frac{\beta_{1}}{\lambda_{1}}-\left(\frac{\sum_{i=1}^{m} a_{i} \eta_{i}+\gamma_{1} T}{\lambda_{1} \lambda_{2}}\right) \beta_{2}-\left(\frac{\delta_{1}}{k^{2} \delta_{3} \lambda_{1}}+\frac{\delta_{2}\left(\sum_{i=1}^{m} a_{i} \eta_{i}+\gamma_{1} T\right)}{k \delta_{3} \lambda_{1} \lambda_{2}}\right) \beta_{3} \\
& +\sum_{i=1}^{m}\left(\frac{\delta_{1} c_{i}}{\delta_{3} \lambda_{1}}-\left(\frac{\sum_{i=1}^{m} a_{i} \eta_{i}+\gamma_{1} T}{\lambda_{1}}\right)\left(\frac{b_{i} k}{\lambda_{2}}-\frac{k \delta_{2} c_{i}}{\delta_{3} \lambda_{2}}\right)-\frac{a_{i}}{\lambda_{1}}\right) \int_{0}^{\eta_{i}} e^{-k\left(\eta_{i}-s\right)} I^{q-1} h(s) d s \\
& +\left(\frac{\delta_{1} \gamma_{3}}{\delta_{3} \lambda_{1}}-\left(\frac{\sum_{i=1}^{m} a_{i} \eta_{i}+\gamma_{1} T}{\lambda_{1}}\right)\left(\frac{\gamma_{2} k}{\lambda_{2}}-\frac{k \delta_{2} \gamma_{3}}{\delta_{3} \lambda_{2}}\right)-\frac{\gamma_{1}}{\lambda_{1}}\right) \int_{0}^{T} e^{-k(T-s)} I^{q-1} h(s) d s
\end{aligned}
$$




$$
\begin{aligned}
& -\sum_{i=1}^{m}\left(\frac{\delta_{1} c_{i}}{k \delta_{3} \lambda_{1}}+\left(\frac{\sum_{i=1}^{m} a_{i} \eta_{i}+\gamma_{1} T}{\lambda_{1}}\right)\left(\frac{\delta_{2} c_{i}}{\delta_{3} \lambda_{2}}-\frac{b_{i}}{\lambda_{2}}\right)\right) I^{q-1} h\left(\eta_{i}\right) \\
& -\left(\frac{\delta_{1} \gamma_{3}}{k \delta_{3} \lambda_{1}}+\left(\frac{\sum_{i=1}^{m} a_{i} \eta_{i}+\gamma_{1} T}{\lambda_{1}}\right)\left(\frac{\delta_{2} \gamma_{3}}{\delta_{3} \lambda_{2}}-\frac{\gamma_{2}}{\lambda_{2}}\right)\right) I^{q-1} h(T) \\
& +\sum_{i=1}^{m}\left(\frac{\delta_{1} c_{i}}{k^{2} \delta_{3} \lambda_{1}}+\frac{\delta_{2} c_{i}\left(\sum_{i=1}^{m} a_{i} \eta_{i}+\gamma_{1} T\right)}{k \delta_{3} \lambda_{1} \lambda_{2}}\right) I^{q-2} h\left(\eta_{i}\right) \\
& +\left(\frac{\delta_{1} \gamma_{3}}{k^{2} \delta_{3} \lambda_{1}}+\frac{\delta_{2} \gamma_{3}\left(\sum_{i=1}^{m} a_{i} \eta_{i}+\gamma_{1} T\right)}{k \delta_{3} \lambda_{1} \lambda_{2}}\right) I^{q-2} h(T), \\
A_{2}= & \frac{\beta_{2}}{\lambda_{2}}+\frac{\delta_{2} \beta_{3}}{k \delta_{3} \lambda_{2}}+\sum_{i=1}^{m}\left(\frac{b_{i} k}{\lambda_{2}}-\frac{k \delta_{2} c_{i}}{\delta_{3} \lambda_{2}}\right) \int_{0}^{\eta_{i}} e^{-k\left(\eta_{i}-s\right)} I^{q-1} h(s) d s \\
& +\left(\frac{\gamma_{2} k}{\lambda_{2}}-\frac{k \delta_{2} \gamma_{3}}{\delta_{3} \lambda_{2}}\right) \int_{0}^{T} e^{-k(T-s)} I^{q-1} h(s) d s+\sum_{i=1}^{m}\left(\frac{\delta_{2} c_{i}}{\delta_{3} \lambda_{2}}-\frac{b_{i}}{\lambda_{2}}\right) I^{q-1} h\left(\eta_{i}\right) \\
& +\left(\frac{\delta_{2} \gamma_{3}}{\delta_{3} \lambda_{2}}-\frac{\gamma_{2}}{\lambda_{2}}\right) I^{q-1} h(T)-\sum_{i=1}^{m} \frac{\delta_{2} c_{i}}{k \delta_{3} \lambda_{2}} I^{q-2} h\left(\eta_{i}\right)-\frac{\delta_{2} \gamma_{3}}{k \delta_{3} \lambda_{2}} I^{q-2} h(T) .
\end{aligned}
$$

By substituting the values of $A_{0}, A_{1}$ and $A_{2}$ in (2.4), we get the desired solution (2.2). The converse of the lemma follows by direct computation. This completes the proof.

\section{Uniqueness result}

Let $\mathcal{P}=\mathrm{C}([0, \mathrm{~T}], \mathbb{R})$ denote the Banach space of all continuous functions from $[0, \mathrm{~T}]$ into $\mathbb{R}$ endowed with the norm defined by $\|u\|=\sup \{|u(t)|, t \in[0, T]\}$.

In view of Lemma 2.5, we transform problem (1.1) into an equivalent fixed point problem as

$$
u=\mathcal{H} u,
$$

where $\mathcal{H}: \mathcal{P} \rightarrow \mathcal{P}$ is defined by

$$
\begin{aligned}
(\mathcal{H} u)(t)= & v_{1}(t)+\int_{0}^{t} e^{-k(t-s)}\left(\int_{0}^{s} \frac{(s-x)^{q-2}}{\Gamma(q-1)} f(x, u(x)) d x\right) d s \\
& +\sum_{i=1}^{m} \omega_{i}(t) \int_{0}^{\eta_{i}} e^{-k\left(\eta_{i}-s\right)}\left(\int_{0}^{s} \frac{(s-x)^{q-2}}{\Gamma(q-1)} f(x, u(x)) d x\right) d s \\
& +v_{2}(t) \int_{0}^{T} e^{-k(T-s)}\left(\int_{0}^{s} \frac{(s-x)^{q-2}}{\Gamma(q-1)} f(x, u(x)) d x\right) d s \\
& +\sum_{i=1}^{m} \psi_{i}(t) \int_{0}^{\eta_{i}} \frac{\left(\eta_{i}-s\right)^{q-2}}{\Gamma(q-1)} f(s, u(s)) d s+v_{3}(t) \int_{0}^{T} \frac{(T-s)^{q-2}}{\Gamma(q-1)} f(s, u(s)) d s \\
& +\sum_{i=1}^{m} \varphi_{i}(t) \int_{0}^{\eta_{i}} \frac{\left(\eta_{i}-s\right)^{q-3}}{\Gamma(q-2)} f(s, u(s)) d s+v_{4}(t) \int_{0}^{T} \frac{(T-s)^{q-3}}{\Gamma(q-2)} f(s, u(s)) d s
\end{aligned}
$$

Observe that problem (1.1) has solutions if the operator equation (3.1) has fixed points.

For computational convenience, we set

$$
\begin{aligned}
\mathrm{Q}= & \sup _{\mathrm{t} \in[0, \mathrm{~T}]}\left\{\frac{\mathrm{t}^{\mathrm{q}-1}\left(1-e^{-k \mathrm{t}}\right)}{k \Gamma(\mathrm{q})}+\frac{\left|\sum_{i=1}^{m} \omega_{i}(\mathrm{t}) \eta_{i}^{\mathrm{q}-1}\left(1-e^{-k \eta_{i}}\right)\right|}{k \Gamma(\mathrm{q})}+\frac{\left|v_{2}(\mathrm{t})\right| \mathrm{T}^{\mathrm{q}-1}\left(1-e^{-k \mathrm{~T}}\right)}{k \Gamma(\mathrm{q})}\right. \\
& \left.+\frac{\left|\sum_{i=1}^{m} \psi_{i}(\mathrm{t}) \eta_{i}^{\mathrm{q}-1}\right|}{\Gamma(\mathrm{q})}+\frac{\left|v_{3}(\mathrm{t})\right| \mathrm{T}^{\mathrm{q}-1}}{\Gamma(\mathrm{q})}+\frac{\left|\sum_{i=1}^{m} \varphi_{i}(\mathrm{t}) \eta_{i}^{\mathrm{q}-2}\right|}{\Gamma(\mathrm{q}-1)}+\frac{\left|v_{4}(\mathrm{t})\right| \mathrm{T}^{\mathrm{q}-2}}{\Gamma(\mathrm{q}-1)}\right\} .
\end{aligned}
$$


Now we are in a position to discuss the existence of a unique solution for the problem (1.1) via Banach's contraction mapping principle.

Theorem 3.1. Assume that $\mathrm{f}:[0, \mathrm{~T}] \times \mathbb{R} \rightarrow \mathbb{R}$ is a continuous function satisfying the Lipschitz condition:

$\left(A_{1}\right)$ there exists a positive number $\ell$ such that $|f(t, u)-f(t, v)| \leqslant \ell|u-v|, \forall t \in[0, T], u, v \in \mathbb{R}$.

Then the boundary value problem (1.1) has a unique solution on $[0, \mathrm{~T}]$ if $\ell<1 / \mathrm{Q}$, where $\mathrm{Q}$ is given by (3.3).

Proof. Consider a set $B_{r}=\{u \in \mathcal{P}:\|u\| \leqslant r\}$, where $r \geqslant \frac{Q M+\left\|v_{1}\right\|}{1-\ell Q}, \sup _{t \in[0, T]}|f(t, 0)|=M$, and $Q, v_{1}$ are respectively given by (3.3) and (2.3). In the first step, we show that $\mathcal{H} B_{r} \subset B_{r}$, where the operator $\mathcal{H}$ is defined by (3.2). For any $u \in B_{r}, t \in[0, T]$, observe that

$$
\begin{aligned}
|f(t, u(t))| & =|f(t, u(t))-f(t, 0)+f(t, 0)| \leqslant|f(t, u(t))-f(t, 0)|+|f(t, 0)| \\
& \leqslant \ell\|u\|+M \leqslant \ell r+M .
\end{aligned}
$$

Then, for $u \in B_{r}$, we obtain

$$
\begin{aligned}
& \|\left(\mathcal{H}(u) \| \leqslant \sup _{t \in[0, T]}\left\{\left|v_{1}(t)\right|+\int_{0}^{t} e^{-k(t-s)}\left(\int_{0}^{s} \frac{(s-x)^{\alpha-2}}{\Gamma(\alpha-1)}|f(x, u(x))| d x\right) d s\right.\right. \\
& +\left|\sum_{i=1}^{m} \omega_{i}(t)\right| \int_{0}^{\eta_{i}} e^{-k\left(\eta_{i}-s\right)}\left(\int_{0}^{s} \frac{(s-x)^{q-2}}{\Gamma(q-1)}|f(x, u(x))| d x\right) d s \\
& +\left|v_{2}(t)\right| \int_{0}^{T} e^{-k(T-s)}\left(\int_{0}^{s} \frac{(s-x)^{q-2}}{\Gamma(q-1)}|f(x, u(x))| d x\right) d s \\
& +\left|\sum_{i=1}^{m} \psi_{i}(t)\right| \int_{0}^{\eta_{i}} \frac{\left(\eta_{i}-s\right)^{q-2}}{\Gamma(q-1)}|f(s, u(s))| d s+\left|v_{3}(t)\right| \int_{0}^{T} \frac{(T-s)^{\alpha-2}}{\Gamma(\alpha-1)}|f(s, u(s))| d s \\
& \left.+\left|\sum_{i=1}^{m} \varphi_{i}(t)\right| \int_{0}^{\eta_{i}} \frac{\left(\eta_{i}-s\right)^{q-3}}{\Gamma(q-2)}|f(s, u(s))| d s+\left|v_{4}(t)\right| \int_{0}^{T} \frac{(T-s)^{q-3}}{\Gamma(q-2)}|f(s, u(s))| d s\right\} \\
& \leqslant(\ell r+M) \sup _{t \in[0, T]}\left\{\int_{0}^{t} e^{-k(t-s)}\left(\int_{0}^{s} \frac{(s-x)^{\alpha-2}}{\Gamma(\alpha-1)} d x\right) d s\right. \\
& +\left|\sum_{i=1}^{m} \omega_{i}(t)\right| \int_{0}^{\eta_{i}} e^{-k\left(\eta_{i}-s\right)}\left(\int_{0}^{s} \frac{(s-x)^{q-2}}{\Gamma(q-1)} d x\right) d s+\left|v_{2}(t)\right| \int_{0}^{T} e^{-k(T-s)}\left(\int_{0}^{s} \frac{(s-x)^{q-2}}{\Gamma(q-1)} d x\right) d s \\
& +\left|\sum_{i=1}^{m} \psi_{i}(t)\right| \int_{0}^{\eta_{i}} \frac{\left(\eta_{i}-s\right)^{q-2}}{\Gamma(q-1)} d s+\left|v_{3}(t)\right| \int_{0}^{T} \frac{(T-s)^{\alpha-2}}{\Gamma(\alpha-1)} d s+\left|\sum_{i=1}^{m} \varphi_{i}(t)\right| \int_{0}^{\eta_{i}} \frac{\left(\eta_{i}-s\right)^{q-3}}{\Gamma(q-2)} d s \\
& \left.+\left|v_{4}(\mathrm{t})\right| \int_{0}^{T} \frac{(\mathrm{T}-\mathrm{s})^{\mathrm{q}-3}}{\Gamma(\mathrm{q}-2)} \mathrm{ds}\right\}+\left\|v_{1}\right\| \\
& \leqslant(\ell r+M) Q+\left\|v_{1}\right\| \leqslant r \text {. }
\end{aligned}
$$

This shows that $\mathcal{H} B_{r} \subset B_{r}$. Next we show that the operator $\mathcal{H}$ is a contraction. Let $u, v \in \mathcal{P}$. Then

$$
\begin{aligned}
& \|\mathcal{H} u-\mathcal{H} v\| \leqslant \sup _{t \in[0, T]}\left\{\int_{0}^{t} e^{-k(t-s)}\left(\int_{0}^{s} \frac{(s-x)^{\alpha-2}}{\Gamma(\alpha-1)}|f(x, u(x))-f(x, v(x))| d x\right) d s\right. \\
& +\left|\sum_{i=1}^{m} \omega_{i}(t)\right| \int_{0}^{\eta_{i}} e^{-k\left(\eta_{i}-s\right)}\left(\int_{0}^{s} \frac{(s-x)^{q-2}}{\Gamma(q-1)}|f(x, u(x))-f(x, v(x))| d x\right) d s \\
& +\left|v_{2}(t)\right| \int_{0}^{T} e^{-k(T-s)}\left(\int_{0}^{s} \frac{(s-x)^{q-2}}{\Gamma(q-1)}|f(x, u(x))-f(x, v(x))| d x\right) d s
\end{aligned}
$$




$$
\begin{aligned}
& +\left|\sum_{i=1}^{m} \psi_{i}(t)\right| \int_{0}^{\eta_{i}} \frac{\left(\eta_{i}-s\right)^{q-2}}{\Gamma(q-1)}|f(s, u(s))-f(s, v(s))| d s \\
& +\left|v_{3}(t)\right| \int_{0}^{T} \frac{(T-s)^{q-2}}{\Gamma(q-1)}|f(s, u(s))-f(s, v(s))| d s \\
& +\left|\sum_{i=1}^{m} \varphi_{i}(t)\right| \int_{0}^{\eta_{i}} \frac{\left(\eta_{i}-s\right)^{q-3}}{\Gamma(q-2)}|f(s, u(s))-f(s, v(s))| d s \\
& \left.+\left|v_{4}(t)\right| \int_{0}^{T} \frac{(T-s)^{q-3}}{\Gamma(q-2)}|f(s, u(s))-f(s, v(s))| d s\right\} \\
& \leqslant \ell|| u-v \| \sup _{t \in[0, T]}\left\{\int_{0}^{t} e^{-k(t-s)}\left(\int_{0}^{s} \frac{(s-x)^{\alpha-2}}{\Gamma(\alpha-1)} d x\right) d s\right. \\
& +\left|\sum_{i=1}^{m} \omega_{i}(t)\right| \int_{0}^{\eta_{i}} e^{-k\left(\eta_{i}-s\right)}\left(\int_{0}^{s} \frac{(s-x)^{q-2}}{\Gamma(q-1)} d x\right) d s+\left|v_{2}(t)\right| \int_{0}^{T} e^{-k(T-s)}\left(\int_{0}^{s} \frac{(s-x)^{q-2}}{\Gamma(q-1)} d x\right) d s \\
& +\left|\sum_{i=1}^{m} \psi_{i}(t)\right| \int_{0}^{\eta_{i}} \frac{\left(\eta_{i}-s\right)^{q-2}}{\Gamma(q-1)} d s+\left|v_{3}(t)\right| \int_{0}^{T} \frac{(T-s)^{\alpha-2}}{\Gamma(\alpha-1)} d s+\left|\sum_{i=1}^{m} \varphi_{i}(t)\right| \int_{0}^{\eta_{i}} \frac{\left(\eta_{i}-s\right)^{q-3}}{\Gamma(q-2)} d s \\
& \left.+\left|v_{4}(t)\right| \int_{0}^{T} \frac{(T-s)^{\alpha-3}}{\Gamma(\alpha-2)} d s\right\} \\
& \leqslant \ell Q\|u-v\|,
\end{aligned}
$$

where we have used (3.3). By the given assumption: $\ell<1 / \mathrm{Q}$, it follows that the operator $\mathcal{H}$ is a contraction. Thus, by Banach's contraction mapping principle, we deduce that the operator $\mathcal{H}$ has a fixed point, which corresponds to a unique solution of the problem (1.1) on $[0, \mathrm{~T}]$.

\section{Existence results}

In this section, we obtain different criteria for the existence of solutions for the problem (1.1) under different constraints on the nonlinearity involved in the problem. The first result relies on Krasnoselskii's fixed point theorem.

Lemma 4.1 (Krasnoselskii's fixed point theorem [17]). Let $Y$ be a closed bounded, convex and nonempty subset of a Banach space $\mathrm{X}$. Let $\mathrm{B}_{1}, \mathrm{~B}_{2}$ be the operators such that

(i) $\mathrm{B}_{1} \mathrm{y}_{1}+\mathrm{B}_{2} \mathrm{y}_{2} \in \mathrm{Y}$ whenever $\mathrm{y}_{1}, \mathrm{y}_{2} \in \mathrm{Y}$;

(ii) $\mathrm{B}_{1}$ is compact and continuous; and

(iii) $\mathrm{B}_{2}$ is a contraction mapping.

Then there exists $z \in \mathrm{Y}$ such that $z=\mathrm{B}_{1} z+\mathrm{B}_{2} z$.

Theorem 4.2. Let $f:[0, T] \times \mathbb{R} \rightarrow \mathbb{R}$ be continuous functions satisfying the condition $\left(A_{1}\right)$ and that $|f(t, x)| \leqslant$ $\mathrm{g}(\mathrm{t}), \quad \forall(\mathrm{t}, \mathrm{x}) \in[0, \mathrm{~T}] \times \mathbb{R}$ with $\mathrm{g} \in \mathrm{C}\left([0, \mathrm{~T}], \mathbb{R}^{+}\right)$, and $\sup _{\mathrm{t} \in[0, \mathrm{~T}]}|\mathrm{g}(\mathrm{t})|=\|\mathrm{g}\|$. In addition, it is assumed that $\ell \mathrm{Q}_{1}<1$, where

$$
\begin{aligned}
\mathrm{Q}_{1}= & \sup _{\mathrm{t} \in[0, \mathrm{~T}]}\left\{\frac{\left|\sum_{i=1}^{m} \omega_{i}(\mathrm{t}) \eta_{i}^{\mathrm{q}-1}\left(1-e^{-k \eta_{i}}\right)\right|}{k \Gamma(\mathrm{q})}+\frac{\left|v_{2}(\mathrm{t})\right| \mathrm{T}^{\mathrm{q}-1}\left(1-\mathrm{e}^{-k \mathrm{~T}}\right)}{k \Gamma(\mathrm{q})}\right. \\
& \left.+\frac{\left|\sum_{i=1}^{m} \psi_{i}(\mathrm{t}) \eta_{i}^{\mathrm{q}-1}\right|}{\Gamma(\mathrm{q})}+\frac{\left|v_{3}(\mathrm{t})\right| \mathrm{T}^{\mathrm{q}-1}}{\Gamma(\mathrm{q})}+\frac{\left|\sum_{i=1}^{\mathrm{m}} \varphi_{i}(\mathrm{t}) \eta_{i}^{\mathrm{q}-2}\right|}{\Gamma(\mathrm{q}-1)}+\frac{\left|v_{4}(\mathrm{t})\right| \mathrm{T}^{\mathrm{q}-2}}{\Gamma(\mathrm{q}-1)}\right\} .
\end{aligned}
$$

Then the problem (1.1) has at least one solution on $[0, \mathrm{~T}]$. 
Proof. Consider $\mathrm{B}_{\overline{\mathrm{r}}}=\{u \in \mathcal{P}:\|u\| \leqslant \overline{\mathrm{r}}\}$, where $\overline{\mathrm{r}} \geqslant \mathrm{Q}\|g\|+\left\|v_{1}\right\|$ and $\mathrm{Q}$ is given by (3.3). Introduce the operators $\mathcal{H}_{1}$ and $\mathcal{H}_{2}$ on $B_{\bar{r}}$ as

$$
\begin{aligned}
\left(\mathcal{H}_{1} u\right)(t)= & \int_{0}^{t} e^{-k(t-s)}\left(\int_{0}^{s} \frac{(s-x)^{\alpha-2}}{\Gamma(\alpha-1)} f(x, u(x)) d x\right) d s \\
\left(\mathcal{H}_{2} u\right)(t)= & v_{1}(t)+\sum_{i=1}^{m} \omega_{i}(t) \int_{0}^{\eta_{i}} e^{-k\left(\eta_{i}-s\right)}\left(\int_{0}^{s} \frac{(s-x)^{q-2}}{\Gamma(q-1)} f(x, u(x)) d x\right) d s \\
& +v_{2}(t) \int_{0}^{T} e^{-k(T-s)}\left(\int_{0}^{s} \frac{(s-x)^{q-2}}{\Gamma(q-1)} f(x, u(x)) d x\right) d s \\
& +\sum_{i=1}^{m} \psi_{i}(t) \int_{0}^{\eta_{i}} \frac{\left(\eta_{i}-s\right)^{q-2}}{\Gamma(q-1)} f(s, u(s)) d s+v_{3}(t) \int_{0}^{T} \frac{(T-s)^{q-2}}{\Gamma(q-1)} f(s, u(s)) d s \\
& +\sum_{i=1}^{m} \varphi_{i}(t) \int_{0}^{\eta_{i}} \frac{\left(\eta_{i}-s\right)^{q-3}}{\Gamma(q-2)} f(s, u(s)) d s+v_{4}(t) \int_{0}^{T} \frac{(T-s)^{q-3}}{\Gamma(q-2)} f(s, u(s)) d s .
\end{aligned}
$$

For $u, v \in B_{\bar{r}}$, it is easy to verify that $\left\|\mathcal{H}_{1} u+\mathcal{H}_{2} v\right\| \leqslant Q\|g\|+\left\|v_{1}\right\|$. Thus, $\mathcal{H}_{1} u+\mathcal{H}_{2} v \in \mathrm{B}_{\bar{r}}$. By using the assumption $\left(A_{1}\right)$ and (4.1), we can get $\left\|\mathcal{H}_{2} u-\mathcal{H}_{2} v\right\| \leqslant \ell Q_{1}\|u-v\|$, which implies that $\mathcal{H}_{2}$ is a contraction in view of the given condition: $\ell Q_{1}<1$.

Notice that continuity of $f$ implies that the operator $\mathcal{H}_{1}$ is continuous. Also, $\mathcal{H}_{1}$ is uniformly bounded on $\mathrm{B}_{\overline{\mathrm{r}}}$ as

$$
\left\|\mathcal{H}_{1} u\right\| \leqslant \frac{\left(1-e^{-k T}\right) T^{q-1}\|g\|}{k \Gamma(q)} .
$$

In the last step, it will be shown that the operator $\mathcal{H}_{1}$ is compact. By fixing $\sup _{(\mathrm{t}, \mathrm{u}) \in[0, \mathrm{~T}] \times \mathrm{B}_{\overline{\mathrm{r}}}}|f(t, u)|=$ $f_{\bar{r}}$ and for $t_{1}, t_{2} \in[0, T]$, we obtain

$$
\begin{aligned}
\mid\left(\mathcal{H}_{1} u\right)\left(t_{2}\right) & -\left(\mathcal{H}_{1} u\right)\left(t_{1}\right) \mid \\
& =f_{\bar{r}}\left|\left(e^{-k t_{2}}-e^{-k t_{1}}\right) \int_{0}^{t_{1}} e^{k s}\left(\int_{0}^{s} \frac{(s-x)^{q-2}}{\Gamma(q-1)} d x\right) d s+\int_{t_{1}}^{t_{2}} e^{-k\left(t_{2}-s\right)}\left(\int_{0}^{s} \frac{(s-x)^{q-2}}{\Gamma(q-1)} d x\right) d s\right| \\
& \leqslant \frac{\left|1-e^{-k\left(t_{2}-t_{1}\right)}\right|}{k \Gamma(q)}\left(\left|t_{1}^{q-1}\left(1-e^{-k t_{1}}\right)\right|+t_{2}^{q-1}\right) f_{\bar{r}} \rightarrow 0 \text { as } t_{2}-t_{1} \rightarrow 0,
\end{aligned}
$$

independently of $u \in B_{\bar{r}}$. This implies that $\mathcal{H}_{1}$ is relatively compact on $B_{\bar{r}}$. Hence, by the Arzelá-Ascoli Theorem, the operator $\mathcal{H}_{1}$ is compact on $B_{\bar{r}}$. Thus all the assumptions of Lemma 4.1 are satisfied. In consequence, the conclusion of Lemma 4.1 is applied and hence the problem (1.1) has at least one solution on $[0, \mathrm{~T}]$.

Our second existence result is based on the following (Schaefer like) fixed point theorem.

Lemma 4.3 ([17]). Let $\mathrm{X}$ be a Banach space. Assume that $\Omega: \mathrm{X} \rightarrow \mathrm{X}$ is a completely continuous operator and the set $\mathrm{Y}=\{\mathrm{u} \in \mathrm{X} \mid \mathrm{u}=\mu \Omega \mathrm{u}, 0<\mu<1\}$ is bounded. Then $\Omega$ has a fixed point in $\mathrm{X}$.

Theorem 4.4. Let $\mathrm{f}:[0, \mathrm{~T}] \times \mathbb{R} \rightarrow \mathbb{R}$ be a continuous function. Assume that there exists a positive constant $\mathrm{L}_{1}$ such that $|\mathrm{f}(\mathrm{t}, \mathrm{u}(\mathrm{t}))| \leqslant \mathrm{L}_{1}$ for $\mathrm{t} \in[0, \mathrm{~T}], \mathrm{u} \in \mathbb{R}$. Then the boundary value problem (1.1) has at least one solution on $[0, T]$.

Proof. In the first step, we show that the operator $\mathcal{H}$ defined by (3.2) is completely continuous. Observe that continuity of $\mathcal{H}$ follows from the continuity of $f$. For a positive constant $r$, let $B_{r}=\{u \in \mathcal{P}:\|u\| \leqslant r\}$ be a bounded ball in $\mathcal{P}$. Then for $t \in[0, T]$, we have

$$
\|(\mathcal{H} u)\| \leqslant \sup _{\mathbf{t} \in[0, \mathrm{~T}]}\left\{\left|v_{1}(\mathrm{t})\right|+\mathrm{L}_{1}\left[\int_{0}^{\mathrm{t}} e^{-\mathrm{k}(\mathrm{t}-\mathrm{s})}\left(\int_{0}^{s} \frac{(\mathrm{s}-\mathrm{x})^{\alpha-2}}{\Gamma(\alpha-1)} \mathrm{d} x\right) \mathrm{ds}\right.\right.
$$




$$
\begin{aligned}
& +\left|\sum_{i=1}^{m} \omega_{i}(t)\right| \int_{0}^{\eta_{i}} e^{-k\left(\eta_{i}-s\right)}\left(\int_{0}^{s} \frac{(s-x)^{q-2}}{\Gamma(q-1)} d x\right) d s \\
& +\left|v_{2}(t)\right| \int_{0}^{T} e^{-k(T-s)}\left(\int_{0}^{s} \frac{(s-x)^{q-2}}{\Gamma(q-1)} d x\right) d s \\
& +\left|\sum_{i=1}^{m} \psi_{i}(t)\right| \int_{0}^{\eta_{i}} \frac{\left(\eta_{i}-s\right)^{q-2}}{\Gamma(q-1)}|f(s, u(s))| d s+\left|v_{3}(t)\right| \int_{0}^{T} \frac{(T-s)^{\alpha-2}}{\Gamma(\alpha-1)} d s \\
& \left.\left.+\left|\sum_{i=1}^{m} \varphi_{i}(t)\right| \int_{0}^{\eta_{i}} \frac{\left(\eta_{i}-s\right)^{q-3}}{\Gamma(q-2)} d s+\left|v_{4}(t)\right| \int_{0}^{T} \frac{(T-s)^{q-3}}{\Gamma(q-2)} d s\right]\right\} \leqslant\left\|v_{1}\right\|+L_{1} Q_{1}
\end{aligned}
$$

where $Q$ is defined by (3.3).

Next we show that the operator $\mathcal{H}$ maps bounded sets into equicontinuous sets of $\mathcal{P}$. Let $\tau_{1}, \tau_{2} \in[0, T]$ with $\tau_{1}<\tau_{2}$ and $u \in B_{r}$. Then we have

$$
\begin{aligned}
\left|(\mathcal{H u})\left(\tau_{2}\right)-(\mathcal{H} u)\left(\tau_{1}\right)\right| \leqslant & \left|\frac{\beta_{3}}{k^{2} \delta_{3}}\left(e^{-k \tau_{2}}-e^{-k \tau_{1}}\right)\right|+\left|\frac{\left(\tau_{2}-\tau_{1}\right)}{\lambda_{2}}\left(\beta_{2}+\frac{\beta_{3} \delta_{2}}{k \delta_{3}}\right)\right| \\
& +\frac{\left|1-e^{-k\left(\tau_{2}-\tau_{1}\right)}\right|}{k \Gamma(q)}\left(\left|\tau_{1}^{q-1}\left(1-e^{-k \tau_{1}}\right)\right|+\tau_{2}^{q-1}\right) \mathrm{L}_{1} \\
& +\sum_{i=1}^{m}\left[\left|\frac{c_{i}\left(e^{-k \tau_{2}}-e^{-k \tau_{1}}\right)}{\delta_{3}}\right|+\left|\frac{k\left(\tau_{2}-\tau_{1}\right)}{\lambda_{2} \delta_{3}}\left(b_{i} \delta_{3}-\delta_{2} c_{i}\right)\right|\right] \frac{L_{1} \eta_{i}^{q-1}}{k \Gamma(q)}\left(1-e^{-k \eta_{i}}\right) \\
& +\left[\left|\frac{\gamma_{3}\left(e^{-k \tau_{2}}-e^{-k \tau_{1}}\right)}{\delta_{3}}\right|+\left|\frac{k\left(\tau_{2}-\tau_{1}\right)}{\lambda_{2} \delta_{3}}\left(\gamma_{2} \delta_{3}-\delta_{2} \gamma_{3}\right)\right|\right] \frac{L_{1} T^{q-1}}{k \Gamma(q)}\left(1-e^{-k T}\right) \\
& +\mid \sum_{i=1}^{m}\left[\left|\frac{c_{i}\left(e^{-k \tau_{2}}-e^{-k \tau_{1}}\right)}{k \delta_{3}}\right|+\left|\frac{\left(\tau_{2}-\tau_{1}\right)}{\lambda_{2} \delta_{3}}\left(b_{i} \delta_{3}-\delta_{2} c_{i}\right)\right|\right] \frac{L_{1} \eta_{i}^{q-1}}{\Gamma(q)} \\
& +\left[\left|\frac{\gamma_{3}\left(e^{-k \tau_{2}}-e^{-k \tau_{1}}\right)}{k \delta_{3}}\right|+\left|\frac{\left(\tau_{2}-\tau_{1}\right)}{\lambda_{2} \delta_{3}}\left(\gamma_{2} \delta_{3}-\delta_{2} \gamma_{3}\right)\right|\right] \frac{L_{1} T^{q-1}}{\Gamma(q)} \\
& +\sum_{i=1}^{m}\left[\left|\frac{c_{i}\left(e^{-k \tau_{2}}-e^{-k \tau_{1}}\right)}{k^{2} \delta_{3}}\right|+\left|\frac{c_{i} \delta_{2}\left(\tau_{2}-\tau_{1}\right)}{k \lambda_{2} \delta_{3}}\right|\right] \frac{L_{1} \eta_{i}^{q-2}}{\Gamma(q-1)} \\
& +\left[\left|\frac{\gamma_{3}\left(e^{-k \tau_{2}}-e^{-k \tau_{1}}\right)}{k^{2} \delta_{3}}\right|+\left|\frac{\gamma_{3} \delta_{2}\left(\tau_{2}-\tau_{1}\right)}{k \lambda_{2} \delta_{3}}\right|\right] \frac{L_{1} T^{q}-2}{\Gamma(q-1)} .
\end{aligned}
$$

As $\tau_{2}-\tau_{1} \rightarrow 0$, the right-hand side of the above inequality tends to zero independently of $u \in B_{r}$. Therefore, by the Arzelá-Ascoli theorem, the operator $\mathcal{H}: \mathcal{P} \rightarrow \mathcal{P}$ is completely continuous.

Finally, we consider the set $\mathrm{V}=\{\mathrm{u} \in \mathcal{P}: u=\mu \mathcal{H} u, 0<\mu<1\}$ and show that $\mathrm{V}$ is bounded. For $u \in V$ and $t \in[0, T]$, we get

$$
\|\mathrm{u}\| \leqslant \mathrm{L}_{1} \mathrm{Q}+\left\|v_{1}\right\| .
$$

Therefore, $\mathrm{V}$ is bounded. Hence, by Lemma 4.3, the problem (1.1) has at least one solution on $[0, \mathrm{~T}]$.

Now we show the existence of solutions for the problem (1.1) via Leray-Schauder nonlinear alternative for single-valued maps.

Lemma 4.5 (Leray-Schauder alternative [11]). Let $\mathrm{E}$ be a Banach space, C a closed, convex subset of E, U an open subset of $\mathrm{C}$ and $0 \in \mathrm{U}$. Suppose that $\mathcal{H}: \overline{\mathrm{U}} \rightarrow \mathrm{C}$ is a continuous, compact (that is, $\mathcal{H}(\overline{\mathrm{U}})$ is a relatively compact subset of C) map. Then either

(i) $\mathcal{H}$ has a fixed point in $\overline{\mathrm{U}}$; or

(ii) there is a $\mathrm{u} \in \mathrm{\partial U}$ (the boundary of $\mathrm{U}$ in $\mathrm{C}$ ) and $\lambda \in(0,1)$ with $\mathrm{u}=\lambda \mathcal{H}(\mathrm{u})$. 
Theorem 4.6. Let $\mathrm{f}:[0, \mathrm{~T}] \times \mathbb{R} \rightarrow \mathbb{R}$ be a continuous function. Assume that

$\left(\mathrm{A}_{3}\right)$ there exist function $\mathrm{p} \in \mathrm{C}\left([0, \mathrm{~T}], \mathbb{R}^{+}\right)$, and nondecreasing function $\Psi: \mathbb{R}^{+} \rightarrow \mathbb{R}^{+}$such that $|\mathrm{f}(\mathrm{t}, \mathrm{u})| \leqslant$ $p(t) \Psi(\|u\|), \quad \forall(t, u) \in[0, T] \times \mathbb{R} ;$

$\left(\mathrm{A}_{4}\right)$ there exists a constant $\bar{M}>0$ such that

$$
\frac{\bar{M}}{\left\|v_{1}\right\|+\|p\| \Psi(\|\bar{M}\|) Q}>1 .
$$

Then the boundary value problem (1.1) has at least one solution on $[0, \mathrm{~T}]$.

Proof. We establish the proof in several steps. Firstly, we show that the operator $\mathcal{H}: \mathcal{P} \rightarrow \mathcal{P}$ defined by (3.2) maps bounded sets into bounded sets in $C([0, T], \mathbb{R})$. For the positive number $r$, let

$$
\mathrm{B}_{\mathrm{r}}=\{\mathrm{u} \in \mathrm{C}([0, \mathrm{~T}], \mathbb{R}):\|\mathrm{u}\| \leqslant \mathrm{r}\}
$$

be a bounded set in $C([0, T], \mathbb{R})$. Then

$$
\begin{aligned}
|\mathcal{H} u(t)| \leqslant & \left|v_{1}(\mathrm{t})\right|+\int_{0}^{t} e^{-k(t-s)}\left(\int_{0}^{s} \frac{(s-x)^{\alpha-2}}{\Gamma(\alpha-1)} p(x) \Psi(\|u\|) d x\right) d s \\
& +\left|\sum_{i=1}^{m} \omega_{i}(t)\right| \int_{0}^{\eta_{i}} e^{-k\left(\eta_{i}-s\right)}\left(\int_{0}^{s} \frac{(s-x)^{q-2}}{\Gamma(q-1)} p(x) \Psi(\|u\|) d x\right) d s \\
& +\left|v_{2}(t)\right| \int_{0}^{T} e^{-k(T-s)}\left(\int_{0}^{s} \frac{(s-x)^{q-2}}{\Gamma(q-1)} p(x) \Psi(\|u\|) d x\right) d s \\
& +\left|\sum_{i=1}^{m} \psi_{i}(t)\right| \int_{0}^{\eta_{i}} \frac{\left(\eta_{i}-s\right)^{q-2}}{\Gamma(q-1)} p(s) \Psi(\|u\|) d s+\left|v_{3}(t)\right| \int_{0}^{T} \frac{(T-s)^{\alpha-2}}{\Gamma(\alpha-1)} p(s) \Psi(\|u\|) d s \\
& +\left|\sum_{i=1}^{m} \varphi_{i}(t)\right| \int_{0}^{\eta_{i}} \frac{\left(\eta_{i}-s\right)^{q-3}}{\Gamma(q-2)} p(s) \Psi(\|u\|) d s+\left|v_{4}(t)\right| \int_{0}^{T} \frac{(T-s)^{q-3}}{\Gamma(q-2)} p(s) \Psi(\|u\|) d s \\
\leqslant & \left\|v_{1}\right\|+\|p\| \Psi(\|u\|) Q,
\end{aligned}
$$

which, on taking the norm for $t \in[0, T]$, yields $\|\mathcal{H} u\| \leqslant\left\|v_{1}\right\|+\|p\| \Psi(r) Q$.

Next we show that $\mathcal{H}$ maps bounded sets into equicontinuous sets of $C([0, T], \mathbb{R})$. Let $t_{1}, t_{2} \in[0, T]$ with $t_{1}<t_{2}$ and $u \in B_{r}$, where $B_{r}$ is a bounded set of $C([0, T], \mathbb{R})$. Then we obtain

$$
\begin{aligned}
\mid \mathcal{H} u\left(t_{2}\right)- & \mathcal{H} u\left(t_{1}\right) \mid \\
\leqslant & \left|v_{1}\left(t_{2}\right)-v_{1}\left(t_{1}\right)\right|+\|p\| \Psi(r)\left[\int_{0}^{t_{1}}\left|e^{-k\left(t_{2}-s\right)}-e^{-k\left(t_{1}-s\right)}\right| \frac{s^{q-1}}{\Gamma(q)} d s+\int_{t_{1}}^{t_{2}} e^{-k\left(t_{2}-s\right)} \frac{s^{q-1}}{\Gamma(q)} d s\right. \\
& +\sum_{i=1}^{m}\left|\omega_{i}\left(t_{2}\right)-\omega_{i}\left(t_{1}\right)\right| \int_{0}^{\eta_{i}} e^{-k\left(\eta_{i}-s\right)} \frac{s^{q-1}}{\Gamma(q)} d s+\left|v_{2}\left(t_{2}\right)-v_{2}\left(t_{1}\right)\right| \int_{0}^{T} e^{-k(T-s)} \frac{s^{q-1}}{\Gamma(q)} d s \\
& +\sum_{i=1}^{m}\left|\psi_{i}\left(t_{2}\right)-\psi_{i}\left(t_{1}\right)\right| \frac{\eta_{i}^{q-1}}{\Gamma(q)}+\left|v_{3}\left(t_{2}\right)-v_{3}\left(t_{1}\right)\right| \frac{T^{q-1}}{\Gamma(q)} \\
& \left.+\sum_{i=1}^{m}\left|\varphi_{i}\left(t_{2}\right)-\varphi_{i}\left(t_{1}\right)\right| \frac{\eta_{i}^{q-2}}{\Gamma(q-1)}+\left|v_{4}\left(t_{2}\right)-v_{4}\left(t_{1}\right)\right| \frac{T^{q-2}}{\Gamma(q-1)}\right] .
\end{aligned}
$$

Obviously the right hand side of the above inequality tends to zero independently of $u \in B_{r}$ as $t_{2}-$ $t_{1} \rightarrow 0$. As $\mathcal{H}$ satisfies the above assumptions, therefore it follows by the Arzelá-Ascoli theorem that $\mathcal{H}: \mathrm{C}([0, \mathrm{~T}], \mathbb{R}) \rightarrow \mathrm{C}([0, \mathrm{~T}], \mathbb{R})$ is completely continuous. 
The result will follow form the Leray-Schauder nonlinear alternative (Lemma 4.5) once we have proved the boundedness of the set of all solutions to the equation $u=\lambda \mathcal{H} u$ for $\lambda \in[0,1]$.

Let $u$ be a solution. Then, for $t \in[0, T]$, and by using the computations in proving that $\mathcal{H}$ is bounded, we have

$$
|u(t)|=\mid \lambda\left(\mathcal{H}(\mathrm{u})(\mathrm{t}) \mid \leqslant\left\|v_{1}\right\|+\|p\| \Psi(\|r\|) \mathrm{Q},\right.
$$

which implies that

$$
\frac{\|u\|}{\left\|v_{1}\right\|+\|p\| \Psi(r) Q} \leqslant 1
$$

In view of $\left(\mathrm{A}_{4}\right)$, there exists $\bar{M}$ such that $\|u\| \neq \bar{M}$. Let us set

$$
\mathrm{U}=\{\mathrm{u} \in \mathrm{C}([0, \mathrm{~T}], \mathbb{R}):\|\mathrm{u}\|<\overline{\mathrm{M}}\} .
$$

Note that the operator $\mathcal{H}: \overline{\mathrm{U}} \rightarrow \mathrm{C}([0, \mathrm{~T}], \mathbb{R})$ is continuous and completely continuous. From the choice of $\mathrm{u}$, there is no $u \in \partial U$ such that $u=\lambda \mathcal{H}(u)$ for some $\lambda \in(0,1)$. Consequently, by the nonlinear alternative of Leray-Schauder type (Lemma 4.5), we deduce that $\mathcal{H}$ has a fixed point $u \in \bar{U}$ which is a solution of the problem (1.1). This completes the proof.

In our final existence result for the problem (1.1), we apply Leray-Schauder degree theory [11].

Theorem 4.7. Let $\mathrm{f}:[0, \mathrm{~T}] \times \mathbb{R} \rightarrow \mathbb{R}$. Assume that there exist constants $0 \leqslant \overline{\mathrm{k}}<\frac{1}{\mathrm{Q}}$, where $\mathrm{Q}$ is given by (3.3) and $\overline{\bar{M}}>0$ such that $|\mathbf{f}(\mathrm{t}, \mathrm{u}(\mathrm{t}))| \leqslant \overline{\mathrm{k}}\|\mathrm{u}\|+\overline{\bar{M}}$ for all $\mathrm{t} \in[0, \mathrm{~T}], \mathrm{u} \in \mathbb{R}$. Then the boundary value problem (1.1) has at least one solution.

Proof. In view of the fixed point problem (3.1), we just need to show that there exists at least one solution $u \in \mathbb{R}$ for (3.1). Define a suitable ball $B_{R} \subset C([0, T]$ with radius $R>0$ as

$$
\mathrm{B}_{\mathrm{R}}=\{\mathrm{u} \in \mathrm{C}([0, \mathrm{~T}]:\|\mathrm{u}\| \leqslant \mathrm{R}\},
$$

where $R$ will be fixed later. Then, it is sufficient to show that $\mathcal{H}: \overline{\mathrm{B}}_{R} \rightarrow \mathrm{C}$ satisfies

$$
\mathrm{u} \neq \lambda \mathcal{H} \mathrm{u}, \forall \mathrm{u} \in \partial \mathrm{B}_{\mathrm{R}} \text { and } \forall \lambda \in[0,1] .
$$

Let us set

$$
\Phi(\lambda, u)=\lambda \mathcal{H} u, \quad u \in C, \quad \lambda \in[0,1] .
$$

Then, by the Arzelá-Ascoli Theorem, $\omega_{\lambda}(\mathfrak{u})=\mathfrak{u}-\Phi(\lambda, u)=\mathfrak{u}-\mathcal{H} u$ is completely continuous. If (4.3) is true, then the following Leray-Schauder degrees are well-defined and by the homotopy invariance of topological degree, it follows that

$$
\begin{aligned}
\operatorname{deg}\left(\omega_{\lambda}, B_{R}, 0\right) & =\operatorname{deg}\left(I-\lambda \mathcal{H}, B_{R}, 0\right)=\operatorname{deg}\left(\omega_{1}, B_{R}, 0\right) \\
& =\operatorname{deg}\left(\omega_{0}, B_{R}, 0\right)=\operatorname{deg}\left(I, B_{R}, 0\right)=1 \neq 0, \quad 0 \in B_{R},
\end{aligned}
$$

where I denotes the unit operator. By the nonzero property of Leray-Schauder degree, $\omega_{1}(t)=u-\lambda \mathcal{H} u=$ 0 for at least one $u \in B_{R}$. To prove (4.3), we assume that $u=\lambda \mathcal{H} u=0$ for some $\lambda \in[0,1]$ and for all $\mathrm{t} \in[0,1]$. Then, as before, one can obtain

$$
|u(t)|=|\lambda(\mathcal{H} u)(t)| \leqslant\left\|v_{1}\right\|+(\bar{k}\|u\|+\overline{\bar{M}}) Q,
$$

which implies that

$$
\|\mathrm{u}\| \leqslant \frac{\left\|v_{1}\right\|+\overline{\bar{M}} \mathrm{Q}}{1-\overline{\mathrm{k}} \mathrm{Q}} .
$$

By letting $R=\frac{\left\|v_{1}\right\|+\overline{\bar{M}} Q}{1-\bar{k} Q}+1,(4.3)$ holds. This completes the proof. 


\section{Examples}

Consider the following anti-periodic multi-point fractional boundary value problem:

$$
\left\{\begin{array}{c}
\left({ }^{c} D^{5 / 2}+2^{c} D^{3 / 2}\right) u(t)=f(t, u(t)), t \in[0,2], \\
u(0)+u(1 / 4)+1 / 2 u(3 / 4)-u(5 / 4)+u(7 / 4)-u(2)=1, \\
u^{\prime}(0)-u^{\prime}(1 / 4)+u^{\prime}(3 / 4)-1 / 2 u^{\prime}(5 / 4)+1 / 4 u^{\prime}(7 / 4)-1 / 2 u^{\prime}(2)=2, \\
1 / 2 u^{\prime \prime}(0)+1 / 4 u^{\prime \prime}(1 / 4)-u^{\prime}(3 / 4)+u^{\prime \prime}(5 / 4)+u^{\prime \prime}(7 / 4)+u^{\prime \prime}(2)=1 .
\end{array}\right.
$$

$\mathrm{T}=2,0<\eta_{1}=1 / 4, \eta_{2}=3 / 4, \eta_{3}=5 / 4, \eta_{4}=7 / 4<\mathrm{T}, \mathrm{k}=2, \alpha_{1}=1, \gamma_{1}=-1, \alpha_{2}=1, \gamma_{2}=-1 / 2, \alpha_{3}=$ $1 / 2, \gamma_{3}=1, a_{1}=1, a_{2}=1 / 2, a_{3}=-1, a_{4}=1, b_{1}=-1, b_{2}=1, b_{3}=-1 / 2, b_{4}=1 / 4, c_{1}=1 / 4, c_{2}=$ $-1, c_{3}=1, c_{4}=1, \beta_{1}=1, \beta_{2}=2, \beta_{3}=1, Q \approx 127.7343, Q_{1} \approx 126.6899$, where $Q$ and $Q_{1}$ are respectively given by (3.3) and (4.1).

- For the applicability of Theorem 3.1 to the problem (5.1), let $f(t, u(t))=\frac{1}{200} \tan ^{-1} u(t)+\operatorname{cost}$ in (5.1). It is easy to find that $\ell=1 / 200$ as $|f(t, u)-f(t, v)| \leqslant \frac{1}{200}|u-v|$ and that $\ell Q \approx 0.6387<1$. Clearly all the conditions of Theorem 3.1 are satisfied. Hence we deduce by the conclusion of Theorem 3.1 that there exists a unique solution for problem (5.1) on $[0,2]$.

- For the illustration of Theorem 4.2, we take $f(t, u(t))=\frac{1}{200} \tan ^{-1} u(t)+\cos t$ in (5.1). Obviously $|f(t, u)| \leqslant \pi / 400+\cos t=g(t),\|g\|=\frac{\pi+400}{400}$ and $\ell_{1} \approx 0.63345<1$. Thus all the conditions of Theorem 4.2 are satisfied. Hence the conclusion of Theorem 4.2 applies to the problem (5.1).

- Let us take $f(t, u(t))=\frac{1}{2} \sin u(t)+e^{-t}+2$ in (5.1) such that $|f(t, u)| \leqslant 7 / 2$. Thus, by the conclusion of Theorem 4.4, we deduce that problem (5.1) has at least one solution on $[0,2]$.

- For the elaboration of Theorem 4.6, let $f(t, u(t))=\frac{1}{\sqrt{t^{2}+4}} \frac{|u|}{1+|u|}$ in (5.1). Clearly $|f(t, u)| \leqslant \frac{1}{\sqrt{t^{2}+4}}$. By fixing $\Psi(\|u\|)=1, p(t)=\frac{1}{\sqrt{t^{2}+4}}$, we find by (4.2) that $\bar{M}>\bar{M}_{1} \approx 90.4603$. In consequence, it follows by the conclusion of Theorem 4.6 that the problem (5.1) has at least one solution on $[0,2]$.

- In order to demonstrate the application of Theorem 4.7, we take $f(t, u(t))=\frac{1}{400} \sin (2 u)+1$ in (5.1) and note that $|f(t, u)| \leqslant\|u\| / 200+1$. Clearly $\bar{k}=\frac{1}{200}, \overline{\bar{M}}=1$ and $0<\bar{k}=\frac{1}{200}<\frac{1}{127.7343}=\frac{1}{Q}$. As all the conditions of Theorem 4.7 hold true, so the conclusion of Theorem 4.7 is applied and hence there exists at least one solution for the problem (5.1) on $[0,2]$.

\section{Concluding remarks}

In this paper, we have discussed the existence and uniqueness of solutions for a new class of boundary value problems consisting of sequential fractional differential equations and a combination of antiperiodic and multi-point boundary conditions by means of appropriate tools of the fixed point theory. Our results are not only new in the given configuration but also correspond to the ones for certain new problems for particular values of the parameters involved in the problem at hand. For example, by taking $a_{i}, b_{i}, c_{i}=0, i=1,2, \ldots, m, \beta_{j}=0, \alpha_{j}=1=\gamma_{j}, j=1,2,3$ in the results of this paper, we obtain the new ones for the problem of sequential fractional differential equations with anti-periodic boundary conditions:

$$
\begin{aligned}
& \left({ }^{c} D^{q}+k^{c} D^{q-1}\right) u(t)=f(t, u(t)), \quad 2<q \leqslant 3, \quad 0<t<T, \\
& u(0)+u(T)=0, \quad u^{\prime}(0)+u^{\prime}(T)=0, \quad u^{\prime \prime}(0)+u^{\prime \prime}(T)=0 .
\end{aligned}
$$

Similarly the results for nonlocal multi-point boundary value problems associated with either left-end point $(x=0)$ or right-end point $(x=T)$ of the interval $[0, T]$ can be obtained by taking $\gamma_{j}=0$ and $\alpha_{j}=0(j=1,2,3)$ respectively in our results. So the present work is a useful contribution to the existing literature on the topic. 


\section{References}

[1] B. Ahmad, J. J. Nieto, Anti-periodic fractional boundary value problems with nonlinear term depending on lower order derivative, Fract. Calc. Appl. Anal., 15 (2012), 451-462. 1

[2] B. Ahmad, J. J. Nieto, Sequential fractional differential equations with three-point boundary conditions, Comput. Math. Appl., 64 (2012), 3046-3052.

[3] B. Ahmad, J. J. Nieto, A class of differential equations of fractional order with multi-point boundary conditions, Georgian Math. J., 21 (2014), 243-248.

[4] B. Ahmad, S. K. Ntouyas, A. Alsaedi, On a coupled system of fractional differential equations with coupled nonlocal and integral boundary conditions, Chaos Solitons Fractals, 83 (2016), 234-241.

[5] S. Aljoudi, B. Ahmad, J. J. Nieto, A. Alsaedi, A coupled system of Hadamard type sequential fractional differential equations with coupled strip conditions, Chaos Solitons Fractals, 91 (2016), 39-46. 1

[6] A. Alsaedi, S. Sivasundaram, B. Ahmad, On the generalization of second order nonlinear anti-periodic boundary value problems, Nonlinear Stud., 16 (2009), 415-420. 1

[7] M. H. Aqlan, A. Alsaedi, B. Ahmad, J. J. Nieto, Existence theory for sequential fractional differential equations with anti-periodic type boundary conditions, Open Math., 14 (2016), 723-735. 1

[8] M. Benchohra, N. Hamidi, J. Henderson, Fractional differential equations with anti-periodic boundary conditions, Numer. Funct. Anal. Optim., 34 (2013), 404-414. 1

[9] A. Bitsadze, A. Samarskii, On some simple generalizations of linear elliptic boundary problems, Soviet Math. Dokl., 10 (1969), 398-400. 1

[10] J.-F. Cao, Q.-G. Yang, Z.-T. Huang, Existence of anti-periodic mild solutions for a class of semilinear fractional differential equations, Commun. Nonlinear Sci. Numer. Simul., 17 (2012), 277-283. 1

[11] A. Granas, J. Dugundji, Fixed point theory, Springer Monographs in Mathematics, Springer-Verlag, New York, (2003). 4.5, 4

[12] J. Jiang, Solvability of anti-periodic boundary value problem for coupled system of fractional p-Laplacian equation, Adv. Difference Equ., 2015 (2015), 11 pages. 1

[13] A. A. Kilbas, H. M. Srivastava, J. J. Trujillo, Theory and applications of fractional differential equations, North-Holland Mathematics Studies, Elsevier Science B.V., Amsterdam, (2006). 2

[14] M. Klimek, Sequential fractional differential equations with Hadamard derivative, Commun. Nonlinear Sci. Numer. Simul., 16 (2011), 4689-4697. 1

[15] Y.-H. Li, A.-B. Qi, Positive solutions for multi-point boundary value problems of fractional differential equations with p-Laplacian, Math. Methods Appl. Sci., 39 (2016), 1425-1434.

[16] L. Peng, Y. Zhou, Bifurcation from interval and positive solutions of the three-point boundary value problem for fractional differential equations, Appl. Math. Comput., 257 (2015), 458-466. 1

[17] D. R. Smart, Fixed point theorems, Cambridge Tracts in Mathematics, Cambridge University Press, London-New York, (1980). 4.1, 4.3

[18] J. Tariboon, T. Sitthiwirattham, S. K. Ntouyas, Existence results for fractional differential inclusions with multi-point and fractional integral boundary conditions, J. Comput. Anal. Appl., 17 (2014), 343-360. 1

[19] J.-R. Wang, Y. Zhou, M. Fečkan, On the nonlocal Cauchy problem for semilinear fractional order evolution equations, Cent. Eur. J. Math., 12 (2014), 911-922.

[20] S.-L. Xie, Y.-M. Xie, Positive solutions of a system for nonlinear singular higher-order fractional differential equations with fractional multi-point boundary conditions, Bound. Value Probl., 2016 (2016), 18 pages. 1

[21] Y. Zhou, Basic theory of fractional differential equations, World Scientific Publishing Co. Pte. Ltd., Hackensack, NJ, (2014). 2 